\title{
Genetic Algorithm in Hill Cipher Encryption
}

\author{
Andysah Putera Utama Siahaan \\ Faculty of Computer Science \\ Universitas Pembangunan Panca Budi \\ J1. Jend. Gatot Subroto Km. 4,5 Sei Sikambing, 20122, Medan, Sumatera Utara, Indonesia
}

\begin{abstract}
The matrix in Hill Cipher was designed to perform encryption and decryption. Every column and row must be inserted by integer numbers. But, not any key that can be given to the matrix used for the process. The wrong determinant result cannot be used in the process because it produces the incorrect plaintext when doing the decryption after the encryption. Genetic algorithms offer the optimized way to determine the key used for encryption and decryption on the Hill Cipher. By determining the evaluation function in the genetic algorithm, the key that fits the composition will be obtained. By implementing this algorithm, the search of the key on the Hill Cipher will be easily done without spending too much time. Genetic algorithms do well if it is combined with Hill Cipher.
\end{abstract}

Keywords - Cryptography, Genetic Algorithm, Hill Cipher

\section{Introduction}

Nowadays, encryption is a very critical aspect to be applied in securing file over the internet. There are two kinds of the cryptography style, such as classic and modern. Hill Cipher is one of the average methods used. It offers the high-security level mechanism. In common use, Hill Cipher has two matrix size, $2 \times 2$ and $3 \times 3$. This research will focus on the $3 \times 3$ matrix. The matrix has nine cells consists of three columns and three rows as well. A random integer number is usually put in each cell. There is a condition achieve where the determinant of the matrix must return 1. It is not easy when it is manually performed. It will take a long time do calculate until it reaches the correct value. The Genetic algorithms try to optimize the time wasted. It generates several or many populations to fit the destination desired. It is expected the cost of time will be minimized. Furthermore, the Genetic algorithms can return the key pairs on how many it is desired but the more keys generated, the more time wasted.

\section{Theories}

The confidential information must be protected from being intercepted [8]. There are various techniques should be used to protect the confidential image data from unauthorized access [7][9]. Hill Cipher is the modulo arithmetic technique in cryptography [1]. Hill Cipher uses the symmetric key as the password to convert plaintext to ciphertext. The Symmetric key is one of the cryptography systems that have the same kind of keys in encryption and decryption. The key employed to encryption is actually distinct from decryption, but they are drawn from the same formula. The key must be inverted before utilized to decrypt the ciphertext. This cryptographic technique has the matrix as the vessel of information exchanges either on encryption or decryption part [2]. The general theory of the matrix used in Hill Cipher is the multiplication between the matrix and the inverse of the matrix [3]. Without getting the right key, the process of encryption and decryption can be done. Figure 1 is the example of encryption process.

Figure 1. Hill Cipher Encryption

$$
\left(\begin{array}{l}
C_{1} \\
C_{2} \\
C_{3}
\end{array}\right)=\left(\begin{array}{lll}
k_{11} & k_{12} & k_{13} \\
k_{21} & k_{22} & k_{23} \\
k_{31} & k_{32} & k_{33}
\end{array}\right)\left(\begin{array}{l}
P_{1} \\
P_{2} \\
P_{3}
\end{array}\right) \operatorname{Mod} 26
$$

$\mathrm{C} 1$ is dependent on the multiplication of $\mathrm{K} 11, \mathrm{~K} 12, \mathrm{~K} 13$ and $\mathrm{P} 1, \mathrm{P} 2, \mathrm{P} 3$. The result of the multiplication has modular expression of the total character. Genetic algorithm is a computational algorithm that inspired the theory of evolution which was later adopted into computational algorithms is used to solve a search value in an 
optimization problem [4]. This algorithm is built on the genetic processes in living organisms. This following figure explains the step of the Genetic algorithm process.

Figure 2. Step of genetic algorithm

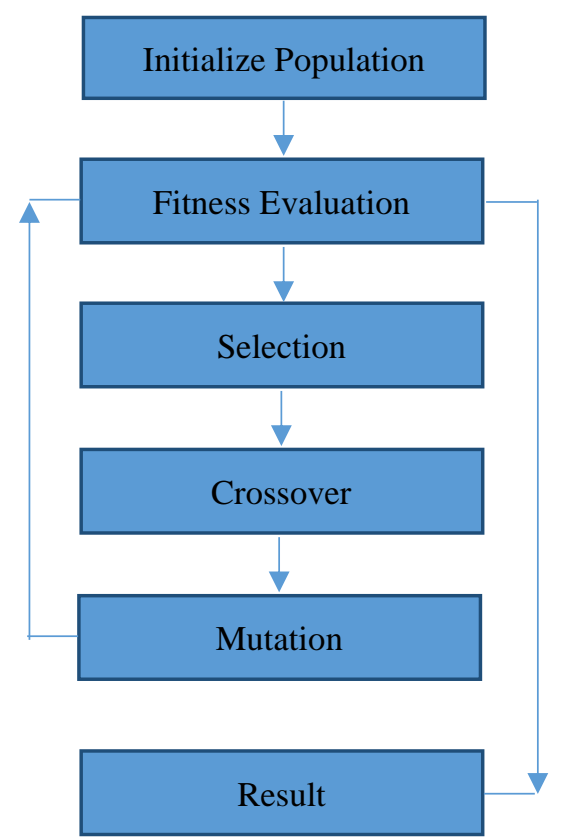

There are three main steps in Genetic algorithms such as selection, crossover and mutation. Selection is used to recombine the population with the highest probability. The random number generated is combined with the cumulative probability. The nearest value is taken in order to replace the original value of the population.

Figure 3. Roulette Wheel Selection

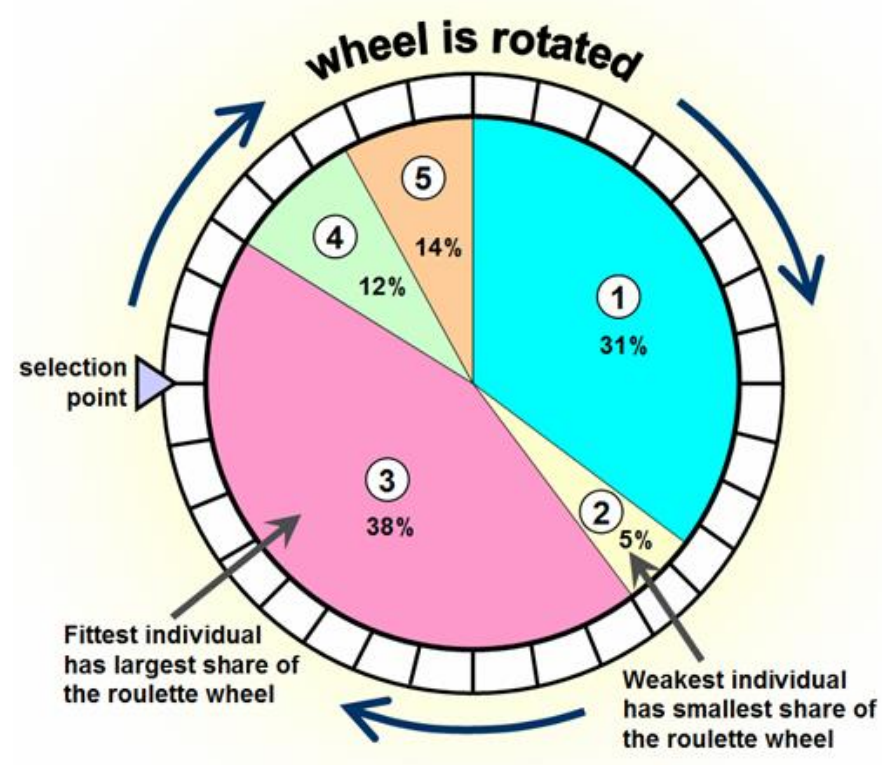

Figure 3 shows one of the selection methods. The method above is Roulette Wheel Selection. Individuals are mapped into a line segment in a sequence such that each individual segment has the exact same size as its fitness. A random number generated. Individuals who have a segment within the segment in the region of the random numbers will be selected [6]. This process is repeated until the number of individuals is obtained. Crossover is a genetic algorithm operator to mix the chromosome with the extra chromosome chosen to produce child chromosome from one generation to the next. It usually selects some qualified parents [5]. The qualification is the crossover rate value. This value relates to select the parent chromosome. 
Figure 4. One cut point crossover

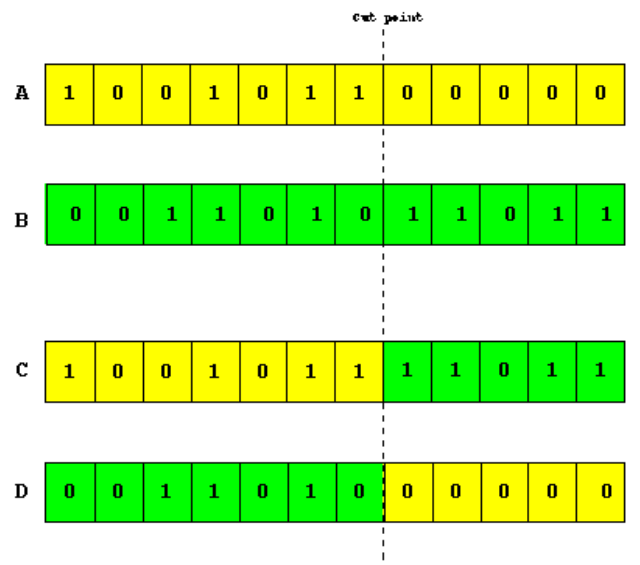

Figure 4 indicates the crossover based on one cut point division. Mutation is a genetic operator employed to maintain genetic diversity from one generation of a population of genetic algorithm chromosomes to the next. This operator repositions the chromosomes by exchanging the value of the chromosome.

Figure 5. Swap Mutation

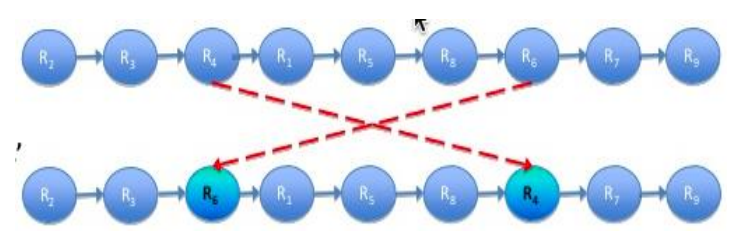

Figure 5 shows the example of swap mutation. The R4 and R6 are swapped in mutation. The chromosome index does not change, but the value of the index is substituted for the other value in the other index. Mutation causes movement in the search space and may produce stronger chromosome.

\section{Related Work}

The previous researches of Hill Cipher do not say anything how to optimize the key search. The key is always obtained by trial and error method. This is not the clever idea to get the correct key in Hill Cipher. It needs the proper determinant to have the cipher text return its original message. It still requires more times to produce the key manually. Once the determinant gets wrong, it has to be recalculated from the beginning. There are nine numbers composed as the key for the encryption and decryption.

\section{Proposed Work}

Every chromosome in Hill Cipher consists of nine numbers. Each cell has a value in range of 0 to 255 which represents the a byte number. Since the ASCII value does not exceed 255, it needs to be nomalized by a modular expression (MOD).

Figure 6. Hill Cipher Chromosome

\begin{tabular}{|c|c|c|c|c|c|c|c|c|}
\hline K11 & K12 & K13 & K21 & K22 & K23 & K31 & K32 & K33 \\
\hline $\mathrm{x}$ & $\mathrm{x}$ & $\mathrm{x}$ & $\mathrm{x}$ & $\mathrm{x}$ & $\mathrm{x}$ & $\mathrm{x}$ & $\mathrm{x}$ & $\mathrm{x}$ \\
\hline
\end{tabular}

Figure 6 shows the form of the Hill Cipher chromosome. The matrix is transformed into one-dimension vector. Each cell is filled with a random integer number (x). The fitness function evaluates the determinant of the chromosome by this following formula.

$$
F=D \text { eterminant }
$$

Where:

$$
\begin{array}{ll}
\mathrm{F} & =\text { Fitness } \\
\mathrm{D} & =\text { Determinant }
\end{array}
$$

The genetic algorithm of this method is rather straightforward than the one of a usual method for scheduling because it does not have to search until the fitness value reaches no error. It searches the ideal fitness which does not contain an even value. Since the determinant is in the odd number, it is good for Hill Cipher. But, in this case, it still find until the determinant reaches 1 . 


\section{Testing and Implementation}

Before knowing how the method works, first thing first is to prepare the data. The first step is to generate the initial population. For example, the Generation is set to 30 and Population Size is 20. The initial random population is given in table 1. Each number in the cell is a random integer.

Table I. Initial Population

\begin{tabular}{|c|c|c|c|c|c|c|c|c|}
\hline K11 & K12 & K13 & K21 & K22 & K23 & K31 & K32 & K33 \\
\hline 108 & 85 & 165 & 89 & 69 & 185 & 97 & 185 & 54 \\
\hline 65 & 86 & 135 & 47 & 224 & 116 & 213 & 112 & 6 \\
\hline 227 & 29 & 41 & 44 & 1 & 141 & 101 & 218 & 32 \\
\hline 174 & 195 & 136 & 196 & 220 & 37 & 108 & 144 & 43 \\
\hline 31 & 232 & 46 & 145 & 120 & 234 & 196 & 242 & 63 \\
\hline 190 & 54 & 140 & 128 & 118 & 179 & 151 & 108 & 43 \\
\hline 90 & 64 & 85 & 24 & 242 & 106 & 154 & 178 & 244 \\
\hline 172 & 124 & 121 & 98 & 194 & 81 & 73 & 183 & 135 \\
\hline 215 & 82 & 163 & 62 & 103 & 13 & 79 & 165 & 164 \\
\hline 147 & 47 & 77 & 212 & 45 & 112 & 249 & 18 & 80 \\
\hline 213 & 7 & 244 & 195 & 246 & 197 & 244 & 25 & 119 \\
\hline 196 & 92 & 115 & 59 & 55 & 190 & 44 & 191 & 27 \\
\hline 134 & 29 & 216 & 72 & 200 & 78 & 196 & 7 & 131 \\
\hline 168 & 214 & 80 & 10 & 104 & 177 & 1 & 114 & 177 \\
\hline 145 & 20 & 91 & 221 & 73 & 79 & 149 & 137 & 73 \\
\hline 50 & 89 & 246 & 142 & 168 & 108 & 85 & 116 & 244 \\
\hline 119 & 141 & 61 & 167 & 254 & 239 & 66 & 77 & 65 \\
\hline 40 & 187 & 243 & 193 & 58 & 195 & 14 & 154 & 172 \\
\hline 190 & 168 & 210 & 137 & 178 & 63 & 5 & 146 & 173 \\
\hline 139 & 26 & 47 & 226 & 179 & 242 & 187 & 137 & 228 \\
\hline
\end{tabular}

Every chromosome is calculated to get the fitness, probability and cumulative probability. The next step is to conduct selection, crossover, and mutation. Table 2 shows the fitness, probability, and cumulative probability of population.

Table II. Fitness, Probability, and Cumulative Probability

\begin{tabular}{|c|c|c|}
\hline $\mathrm{F}$ & $\mathrm{P}$ & $\mathrm{CP}$ \\
\hline 1 & 0,0003652 & 0,0003652 \\
\hline 64 & 0,0233747 & 0,02374 \\
\hline 210 & 0,0766983 & 0,1004383 \\
\hline 238 & 0,0869248 & 0,187363 \\
\hline 9 & 0,0032871 & 0,1906501 \\
\hline 76 & 0,0277575 & 0,2184076 \\
\hline 150 & 0,0547845 & 0,2731921 \\
\hline 186 & 0,0679328 & 0,3411249 \\
\hline 203 & 0,0741417 & 0,4152666 \\
\hline 174 & 0,06355 & 0,4788167 \\
\hline 241 & 0,0880205 & 0,5668371 \\
\hline 84 & 0,0306793 & 0,5975164 \\
\hline 0 & 0 & 0,5975164 \\
\hline 152 & 0,055515 & 0,6530314 \\
\hline 172 & 0,0628196 & 0,715851 \\
\hline 188 & 0,0686633 & 0,7845142 \\
\hline 223 & 0,0814463 & 0,8659606 \\
\hline 169 & 0,0617239 & 0,9276844 \\
\hline 2 & 0,0007305 & 0,9284149 \\
\hline 196 & 0,0715851 & 1 \\
\hline & & \\
\hline
\end{tabular}


The process of selection, crossover and mutation has modified the population structure. The order of the key changes to the other number from other chromosomes. The new generation results from the latest updated chromosomes as saw in Table 2 .

Table III. Next Generation Population

\begin{tabular}{|c|c|c|c|c|c|c|c|c|}
\hline K11 & K12 & K13 & K21 & K22 & K23 & K31 & K32 & K33 \\
\hline 134 & 47 & 216 & 112 & 213 & 90 & 18 & 7 & 131 \\
\hline 85 & 47 & 244 & 195 & 85 & 212 & 244 & 196 & 249 \\
\hline 119 & 63 & 77 & 78 & 145 & 72 & 249 & 18 & 135 \\
\hline 108 & 47 & 197 & 179 & 47 & 239 & 119 & 234 & 65 \\
\hline 167 & 89 & 7 & 142 & 179 & 108 & 200 & 116 & 89 \\
\hline 78 & 64 & 116 & 24 & 242 & 112 & 27 & 191 & 80 \\
\hline 80 & 44 & 185 & 7 & 1 & 141 & 62 & 242 & 32 \\
\hline 254 & 29 & 106 & 196 & 200 & 50 & 13 & 29 & 185 \\
\hline 31 & 232 & 46 & 145 & 120 & 216 & 154 & 178 & 244 \\
\hline 139 & 85 & 227 & 226 & 212 & 165 & 168 & 137 & 228 \\
\hline 218 & 244 & 41 & 163 & 168 & 147 & 112 & 242 & 244 \\
\hline 228 & 85 & 147 & 234 & 69 & 97 & 131 & 141 & 54 \\
\hline 147 & 47 & 77 & 61 & 232 & 246 & 249 & 18 & 80 \\
\hline 215 & 82 & 142 & 101 & 103 & 29 & 79 & 165 & 164 \\
\hline 187 & 120 & 86 & 59 & 55 & 190 & 44 & 6 & 213 \\
\hline 31 & 45 & 46 & 134 & 116 & 89 & 196 & 242 & 63 \\
\hline 65 & 86 & 246 & 196 & 224 & 92 & 66 & 112 & 72 \\
\hline 77 & 115 & 135 & 246 & 224 & 26 & 213 & 112 & 6 \\
\hline 116 & 196 & 65 & 77 & 45 & 212 & 25 & 137 & 50 \\
\hline 139 & 26 & 47 & 226 & 45 & 47 & 187 & 242 & 108 \\
\hline & & & & & & & & \\
\hline
\end{tabular}

This process continues to the latest generation. It is the last result where the Hill Cipher keys are generated. At the end of the process to the total of the Hill Cipher key is variant. Table 4 shows the final result of the Genetic algorithm.

Table IV. The Final Result

\begin{tabular}{|c|c|c|c|c|c|c|c|c|}
\hline K11 & K12 & K13 & K21 & K22 & K23 & K31 & K32 & K33 \\
\hline 147 & 69 & 62 & 147 & 232 & 82 & 29 & 147 & 147 \\
\hline 147 & 62 & 147 & 232 & 246 & 29 & 82 & 147 & 246 \\
\hline 82 & 147 & 232 & 69 & 246 & 82 & 246 & 72 & 147 \\
\hline
\end{tabular}

After getting the result, the key combination is now formed as showed in Table 5. The numbers showed in the blocks are generated by the genetic algorithm. It is cannot be performed manually because it takes much time to calculate the determinant.

Table V. The Key Combination

\begin{tabular}{|c|c|c|c|c|c|c|c|c|}
\hline \multicolumn{3}{|c|}{ Key 1} & \multicolumn{3}{c|}{ Key 2} & \multicolumn{3}{c|}{ Key 3 } \\
\hline 147 & 69 & 62 & 147 & 62 & 147 & 82 & 147 & 232 \\
\hline 147 & 232 & 82 & 232 & 246 & 29 & 69 & 246 & 82 \\
\hline 29 & 147 & 147 & 82 & 147 & 246 & 246 & 72 & 147 \\
\hline
\end{tabular}

The key in the block section is used to encrypt the plaintext in Hill Cipher algorithm.

Plaintext $\quad: \quad$ ANDYSAHXX $\quad\left(\begin{array}{lll}65 & 78 & 68 \\ 89 & 83 & 65 \\ 72 & 88 & 88\end{array}\right)$ 


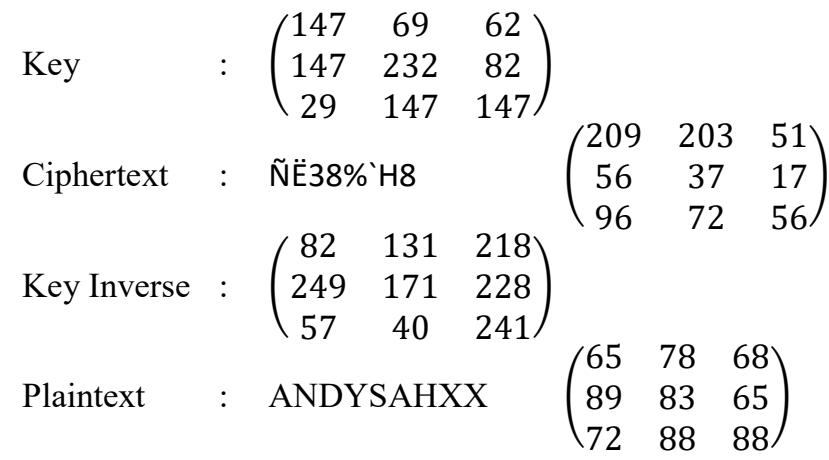

There are three keys produced by the genetic algorithm. The calculation aboe is the proof of Key 1 . The determinant is 1 and it is suitable for the Hill Cipher process. When the determinant is 1 , it can return the ciphertext back to the plain text.

\section{Conclusion}

On the Hill Cipher algorithm that uses a $3 \times 3$ matrix, searching key that has the proper determinant takes time. If it is performed in that way, it slows down the process of cryptography. Genetic algorithms greatly assist the process of the encryption and the decryption on the Hill Cipher. It produces a series of numbers quickly. This technique generates several alternatives that can be used on the Hill Cipher algorithm. In this study, the genetic algorithm has a valuable contribution when combined with the Hill Cipher method.

\section{References}

[1] Abdullah, A. A., Khalaf, R., \& Riza, M. (2015). A Realizable Quantum Three-Pass Protocol Authentication. Mathematical Problems in Engineering.

[2] Chase, J., \& Davis, M. (2010). Extending the Hill Cipher.

[3] Chowdhury, S. I., Shohag, S. A., \& Sahid, H. (2011). A Secured Message Transaction Approach by Dynamic Hill Cipher Generation and Digest Concatenation. International Journal of Computer Applications, 23(9), 25-31.

[4] Ghanbari, A. A., Broumandnia, A., Navidi, H., \& Ahmadi, A. (2012). Brain Computer Interface with Genetic Algorithm. International Journal of Information and Communication Technology Research, 2(1), 79-86.

[5] Lin, C. H., Yu, J. L., Liu, J. C., Lai, W. S., \& Ho, C. H. (2009). Genetic Algorithm for Shortest Driving Time in Intelligent Transportation Systems. International Journal of Hybrid Information Technology, 2(1), 21-30.

[6] Szénási, S., \& Vámossy, Z. (2013). Implementation of a Distributed Genetic Algorithm for Parameter Optimization in a Cell Nuclei Detection Project. Acta Polytechnica Hungarica, 10(4), 59-86.

[7] A. P. U. Siahaan, "RC4 Technique in Visual Cryptography RGB Image Encryption," International Journal of Computer Science and Engineering, vol. 3, no. 7, pp. 1-6, 2016

[8] A. P. U. Siahaan, “Three-Pass Protocol Concept in Hill Cipher Encryption Technique," International Journal of Science and Research, vol. 5, no. 7, 2016.

[9] A. P. U. Siahaan, "Factorization Hack of RSA Secret Numbers," International Journal of Engineering Trends and Technology, vol. 37 , no. 1 , pp. $15-18,2016$.

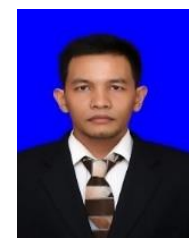

\section{Biography}

Andysah Putera Utama Siahaan was born in Medan, Indonesia, in 1980. He received the S.Kom. degree in computer science from Universitas Pembangunan Panca Budi, Medan, Indonesia, in 2010, and the M.Kom. in computer science as well from the University of Sumatera Utara, Medan, Indonesia, in 2012. In 2010, he joined the Department of Engineering, Universitas Pembangunan Panca Budi, as a Lecturer, and in 2012 became a junior researcher. He is applying for his $\mathrm{Ph}$. D. degree in 2016. He has written in several international journal and conference. He is now active in writing papers and joining conferences. 